Original paper

\title{
The scientific publications of AIFM members in 2015-2019: A survey of the FutuRuS working group
}

\author{
Lorenzo Placidi $^{\mathrm{a}, 1}$, Roberta Castriconi ${ }^{\mathrm{b}, 1}{ }$, Tiziana Rancati ${ }^{\mathrm{c}}$, Michela Lecchi ${ }^{\mathrm{d}}$, Franco Fusi ${ }^{\mathrm{e}}$, \\ Paolo Russo $^{\mathrm{f}}$, Carlo Cavedon ${ }^{\mathrm{g}}$, Claudio Fiorino ${ }^{\mathrm{b}, 2}{ }^{\text {, }}$, Cristina Garibaldi ${ }^{\mathrm{h},{ }^{*}, 2}$ \\ ${ }^{\text {a }}$ Fondazione Policlinico Universitario A. Gemelli IRCCS, Roma, Italy \\ ${ }^{\mathrm{b}}$ Medical Physics, San Raffaele Scientific Institute, Milano, Italy \\ ${ }^{\mathrm{c}}$ Prostate Cancer Program, Fondazione IRCCS Istituto Nazionale dei Tumori, Milano, Italy \\ ${ }^{\mathrm{d}}$ Health Physics Unit, ASST Santi Paolo e Carlo, Milan, Italy \\ e Department of Experimental and Clinical Biomedical Sciences "Mario Serio", University of Florence, Florence, Italy \\ ${ }^{\mathrm{f}}$ Università di Napoli Federico II, Dipartimento di Fisica "Ettore Pancini”, Napoli, Italy \\ ${ }^{\mathrm{g}}$ Medical Physics Unit, Azienda Ospedaliera Universitaria Integrata - Verona, Italy \\ ${ }^{\mathrm{h}}$ Unit of Radiation Research, IEO European Institute of Oncology, IRCCS, Milano, Italy
}

\section{A R T I C L E I N F O}

\section{Keywords:}

Scientific publications

Medical physicists

Radiation oncology

Radiology

Nuclear medicine

\begin{abstract}
A B S T R A C T
Purpose: Within the Italian Association of Medical Physics and Health Physics (AIFM) working group "FutuRuS" we carried out a survey regarding the number of the peer-reviewed articles by AIFM members.

Methods: We surveyed papers published in the years 2015-2019. Data extracted from Scopus included information regarding authors, title, journal, impact factor (IF), leading or standard authorship by AIFM members, keywords, type of collaboration (monocentric/multicentric/international), area of interest [radiation oncology (RO), radiology (RAD), nuclear medicine (NM), radioprotection (RP) and professional issue (PI)] and topics. Results: We found 1210 papers published in peer-reviewed journals: 48\%, 22\%, 16\%, 6\%, 2 and 6\% in RO, RAD, NM, RP, PI and other topics, respectively. Forty-seven percent of the papers involved monocentric teams, 31\% multicentric and $22 \%$ international collaborations. Leading authorship of AIFM members was in $56 \%$ of papers, with a corresponding IF equal to $52 \%$ of the total IF $\left(3342, \mathrm{IF}_{\text {mean }}=2.8, \mathrm{IF}_{\max }=35.4\right)$. The most represented journal was Physica Medica, with 15\% of papers, while a relevant fraction of IF (54\%) appeared in clinically oriented journals. The number of papers increased significantly between 2015 and 2016 and remained almost constant in 2017-2019.

Conclusions: This survey led to the first quantitative assessment of the number and theme distribution of peerreviewed scientific articles contributed by AIFM members. It constitutes a ground basis to support future AIFM strategies and promote working groups on scientific activity of medical physicists, and to build the basis for rational comparison with other countries, first of all within Europe.
\end{abstract}

\section{Introduction}

Medical physicists have a distinctive role as health professionals, mostly in radiation oncology (RO), radiology (RAD) and nuclear medicine (NM), collaborating with physicians and other health professionals in diagnosis, treatment and rehabilitation of human diseases, by developing and implementing medical technologies, and by assessing and promoting high quality medical care for patients. As regards diagnostic and therapeutic use of ionizing radiation, medical physicists play a key role in patient radiation safety, but also non-ionizing radiation medical applications is an active field of research for medical physicists [1]. It is widely recognized that while the clinical service is a fundamental part of the medical physics profession, the roadmap to its advancement is implemented via scientific research: without scientific and technological research and development, the medical physics profession has no future. The International Standard Classification of Occupations (ISCO) of the

\footnotetext{
* Corresponding author.

E-mail address: cristina.garibaldi@ieo.it (C. Garibaldi).

1 Co-first authors.

2 Co-last authors.
} 
International Labour Organization (ILO) classifies the Medical Physicist profession under group 211 "Physicists and Astronomers" [2] and a specific task for this class is "ensuring the safe and effective delivery of radiation (ionising and non-ionising) to patients to achieve a diagnostic or therapeutic result as prescribed by a medical practitioner" [3]. The International Organization of Medical Physics (IOMP) identifies Medical Physicists as those professionals who "apply knowledge and methodology of science of physics to all aspects of medicine, to conduct research, develop or improve theories and address problems" in healthcare [4].

The emergence and continuous evolution of advanced medical technologies over the last decades has significantly expanded the scope and responsibilities of medical physicists [5-7]. In the field of RO, the safe and optimal implementation of intensity modulated radiotherapy, image-guidance, innovative tools for plan optimization (including automating planning), adaptive radiotherapy, heavy particle radiotherapy, MR-guided machines and artificial intelligence (AI) tools implementation were largely driven by medical physics research [8-13].

A steadily increasing recognition of the professional status of the medical physics experts (MPE), reinforced by the EC Council Directive 2013/59/EURATOM [14], introduced in the European Union and recently implemented in the Italian law, has led to a positive evolution of the MPE profession, with continuously increased reputation. Importantly, the new directive clearly defined the responsibilities of the MPE and the obligation for the Member States to ensure their training and recognition as healthcare professionals. Indeed, apart from their clinical service, medical physicists have well-established scientific and research roles from cutting edge research to translational research, technology innovation up to clinical implementation, not always formally recognized [15]. The medical physicists' scientific and research activities represent a strong and well assessed strategic component of the advancement of medicine, in particular of RO, RAD and NM; this seems to be even more important nowadays, if considering the increasing integration of multidisciplinary knowledge and translational research which is required in all these fields.

In the last years, a lively debate within the medical physicists' international community is underway on the need to promote actions and implement policies to reinforce the scientific aspects of medical physics in synergy with the professional aspects [16-18]. International task groups have been established in the US within the American Association of Medical Physicists (AAPM) in 2010 [19] and more recently in Europe within the European Society for Radiotherapy and Oncology (ESTRO) [16-18] to reinforce the scientific profile of the MPE in synergy with their professional role. Of note, the ESTRO group on "Future of Medical Physicists in Radiation Oncology", in collaboration with the AAPM working group on "Future of Medical Physics Research and Academic Training" (WGFRMP), has recently identified priorities for the future grand challenges for medical physics in RO [20]. AAPM WGFRMP performs "... activities to secure sustainable growth and improvement in the long-term future environment for high quality research and academic training of physicists in medicine" [19].

In 2019 EFOMP and ESTRO setup a joint Physics Committee Working Group for review the Core Curriculum in medical physics in radiation oncology, and also to reinforce the scientific role of the medical physicists [21].

A working group was established within the Italian Association of Medical Physics and Health Physics (AIFM), a scientific and professional society with more than 1200 members (year 2020 registry), devoted to promote, develop and coordinate professional, scientific and research activities of physics applied to medicine. In large majority, the members are MPE working exclusively in public/private hospitals (64\%), 4\% are academic staff or members of public research institutions, $11 \%$ are medical physics residents (i.e. university students of Specialization Schools in Medical Physics present in many public and private universities in Italy) and $20 \%$ are freelancers or employed in industries. The AIFM working group on the "Future on medical physics and the need to reinforce its scientific role" (FutuRuS) was established in 2017 to promote initiatives to strengthen the synergy between professional and scientific roles of MPE in a unified approach. The group, involving more than 75 members, has put in place several initiatives to promote within AIFM members the importance of the scientific activity, involving both education/training areas and the professional level. FutuRuS also contributed within AIFM in supporting policies and initiatives aimed to emphasize the relevance of the medical physics scientific production and its visibility, to increase the scientific collaboration between universities and hospitals, and to promote the scientific activity of MPs in "out-offield" environments, also through the exploration of new (and multidisciplinary) fields of interest. Nevertheless, a quantitative analysis on the scientific production of the MPs, either in Italy or in other countries, that could support the visibility of the MPs scientific activity, has not been performed so far.

Within this context, the FutuRuS group decided to carry out a survey regarding the scientific publications of AIFM members during the period 2015-2019, grouped in the major domains of RO, RAD, NM, radioprotection (RP) and professional issues (PI). Other less common areas of interest of MPE outside radiological practices, such as non-ionizing radiation, physiology and audiology, were categorized as "other". This analysis aimed to: 1) highlight the topics of major interest for AIFM members; 2) generate a first quantitative evaluation for future "trend" investigations; 3) build the basis for rational comparison with other countries, first of all within Europe.

\section{Materials and methods}

The Elsevier's abstract and citation database Scopus was interrogated using the Scopus Author function. The search covered the period from 2015 to 2019 and included all directors of Medical Physics Departments in Italy, hospital medical physicists with a substantial scientific production and academic medical physicists, members of AIFM. For each paper the following information were retrieved and organized in a database: full authors list, affiliations, title, journal, Digital Object Identifier (DOI), and keywords. Information from Scopus was further used to classify papers based on: (i) authorship for AIFM members ("leading authorship" including at least one AIFM author as first/last/ corresponding author- vs "standard authorship"), (ii) total and leading impact factor (IF) (leading IF is computed considering only the papers with the authorship), (iii) type of collaboration("monocentric" vs "national multicentric" vs "international"), (iv) area of interest (RO, NM, RAD, RP, PI, other) and the relative ( $v)$ topics.

Descriptive statistics was used to analyse distribution of publications per year, journal, area of interest, form of authorship, collaborative group, total and leading IF. Further sub-group analysis, such as geographical international collaborative distribution, percentage of the leading authorship by topic for the international collaboration and number of the publications by area of interest per year have been performed to improve the granularity description of the scientific production. A p-value less than 0.05 was considered statistically significant.

\section{Results}

According to the search criteria, 1210 scientific articles were found. The number of publications per year was approximately a constant over the four-year period 2016-2019 (around 250/y). We found a statistically significant increase in the number of publications (about 5\%, $p<$ 0.0001) from 2015 to 2016, as shown in Fig. 1a.

Fig. $1 \mathrm{~b}$ reports the distribution of papers with respect to the area of interest. Fig. 2 shows the number of publications by area of interest as a function of time. RO presented a not statistically significant peak in the number of articles in 2017, with 135 articles $(p=0.17)$ and an average number of publications per year of 116 , while the minimum number of publications was 86, achieved in year $2015(p<0.0001)$. On the other hand, RAD reached the maximum number of publications by AIFM members in $2018(77, p=0.02)$ with an average number of publications 

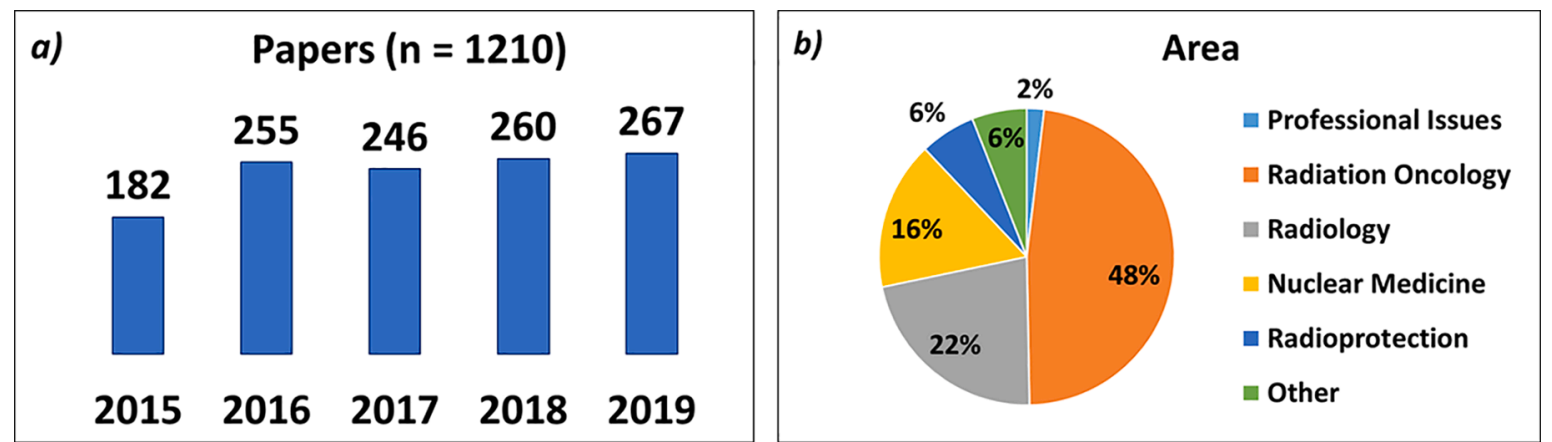

Fig. 1. Number of published papers per year (a) and percentage of the publications' area of interest (b), for scientific papers of AIFM members in the years 2015-2019.

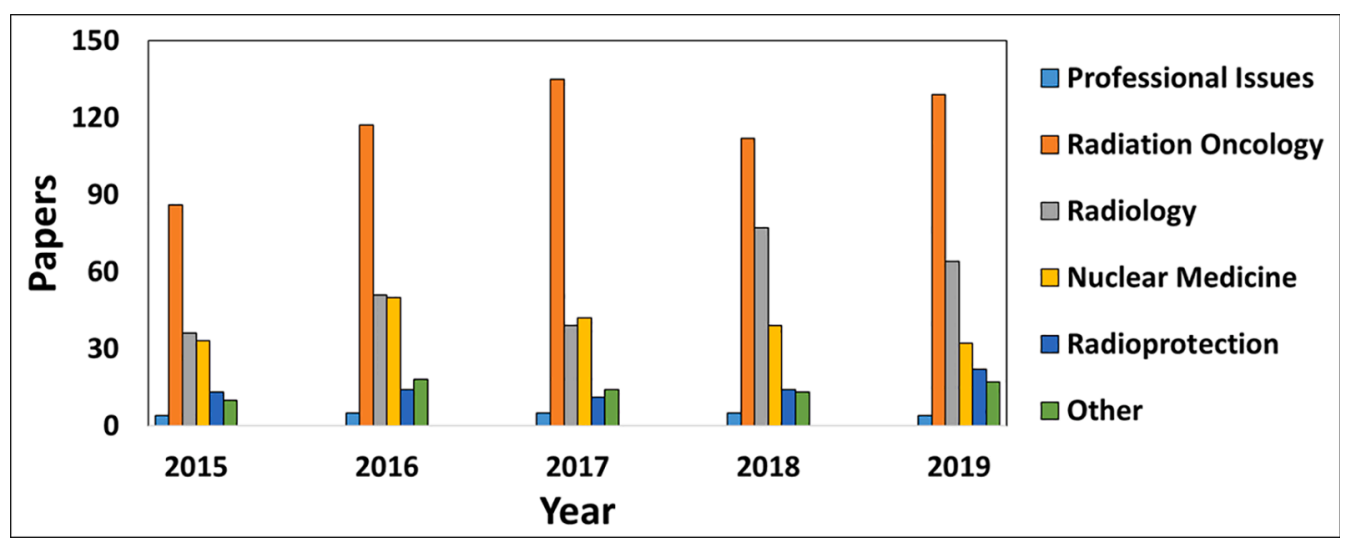

Fig. 2. Number of published articles by area of interest per year, by AIFM members.

per year equal to 53. NM showed an almost constant number of publications per year, reaching a maximum number of 50 (not statistically significant, $p=0.18$ ) in 2016, with an average publication per year of 39. The areas PI, RP and Other had a much lower average number of publications per year equal to 14,4 and 14, respectively.

Table 1 presents the topics grouped in the different areas of interest. Concerning RO, the higher percentage of scientific publications were on modelling-clinical (27\%), particle therapy (22\%), dosimetry and QA $(20 \%)$ and photon and high-energy electron treatment (19\%) topics. In $\mathrm{RAD}$, the higher percentage of publications were on dosimetry and QA ( $41 \%$ ), image quality (32\%), quantitative imaging/radiomics (21\%) and big data/AI/automation (6\%). In $\mathrm{NM}$, the higher percentage of

Table 1

Percentage of the scientific publications for each topic of the corresponding area of interest.

\begin{tabular}{lll}
\hline Area & Topic & $\begin{array}{l}\text { Publications } \\
(\%)\end{array}$ \\
\hline Radiation & Big data/AI/Automation & 1 \\
Oncology & Brachytherapy & 4 \\
& IGRT/ART & 7 \\
& Modelling-clinical & 27 \\
& Particle therapy & 22 \\
& Dosimetry and QA & 20 \\
& Photon and high-energy electron & 19 \\
& treatment & 6 \\
& Big data/AI/Automation & 21 \\
& Quantitative imaging/radiomics & 32 \\
& Image quality & 41 \\
& Dosimetry and QA & 26 \\
Nuclear Medicine & Conventional NM & 30 \\
& NM therapy and dosimetry & 32 \\
& PET & 12 \\
\hline
\end{tabular}

publications were on PET (32\%), NM therapy and dosimetry (30\%) and conventional NM (26\%).

Fig. S1 in Supplementary material shows the number of scientific papers by topic per year, exclusively for the three main area of interest: RO (Figure S1a), RAD (Figure S1b), and NM (Figure S1c).

Concerning papers in the RAD topics evaluated over the years, the number of items in the dosimetry and QA topics doubled in 2018 and papers focused on image quality slightly increased in number over the years in 2015-2019. In the NM area of interest, a constant increasing number of publications for the PET topics was observed over the years, even though in 2019 the number of articles decreased by one third with respect to 2018: in this year the number of published articles reached a maximum.

As far as authorship is concerned, leading authorship was found in $56 \%$ of the publications. Regarding the type of collaboration, $47 \%$ of the papers were monocentric, $31 \%$ national-multicentric and $22 \%$ international.

Fig. 3 shows the number and the worldwide localization of the international collaborations. Among them, 77\% were within European institutions/teams, $13 \%$ with North and South America, 5\% with Asia, $4 \%$ with Oceania and $1 \%$ with Africa. The highest number of publications from international collaborations included institutions/groups from Germany (68), UK (62), USA (57) and Switzerland (54).

The percentage of the leading authorship by topic for the international collaborations is shown in Figure S2 (supplementary materials). The highest percentages (greater than60\%) were found in the fields of dosimetry and QA, photon and high-energy electron treatment techniques, IGRT/ART, brachytherapy for RO and radio-pharmacy and radiobiology for NM.

Fig. 4 summarizes the total and leading IF over the last 5 years. The total $\mathrm{IF}$ was $3341.9\left(\mathrm{IF}_{\text {mean }}=2.8, \mathrm{IF}_{\max }=35.4\right)$, while the leading $\mathrm{IF}$ was 1736.7. The time trend of the IF reflected the one of the number of 


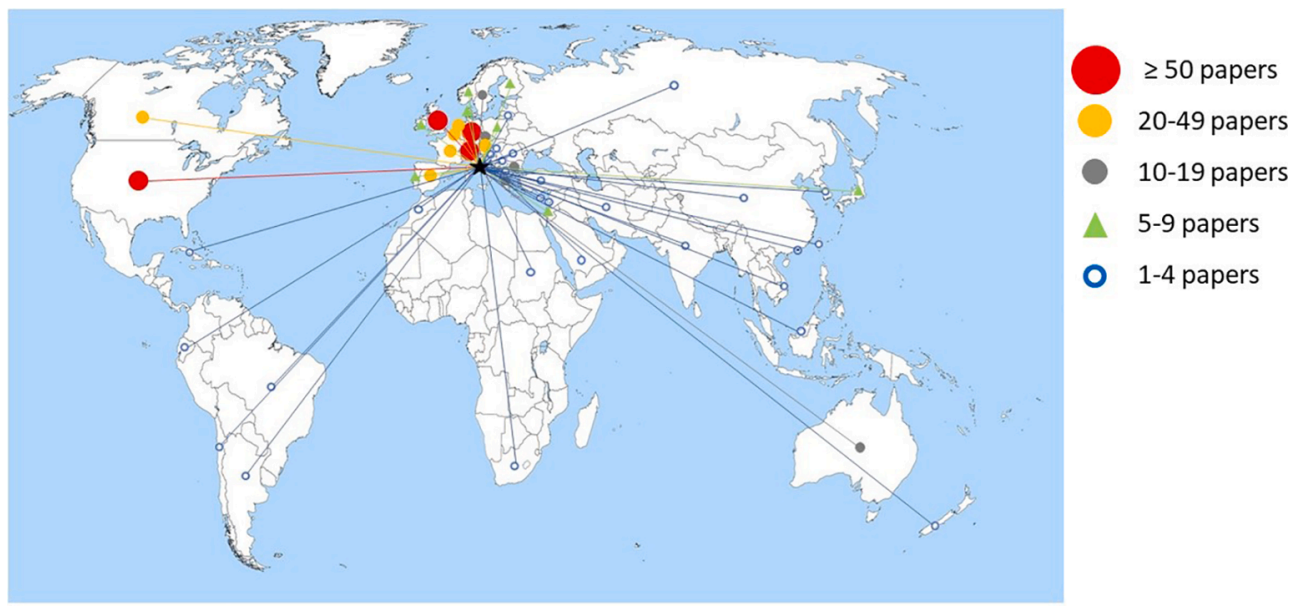

Fig. 3. Number and localization of the international collaboration publications. Red dots identify international collaboration with more than 50 published papers, yellow dots between 20 and 49 published papers, grey dots between 10 and 19 published papers, green triangles between 5 and 9 published papers and blue circles between 1 and 4 published papers. (For interpretation of the references to colour in this figure legend, the reader is referred to the web version of this article.)

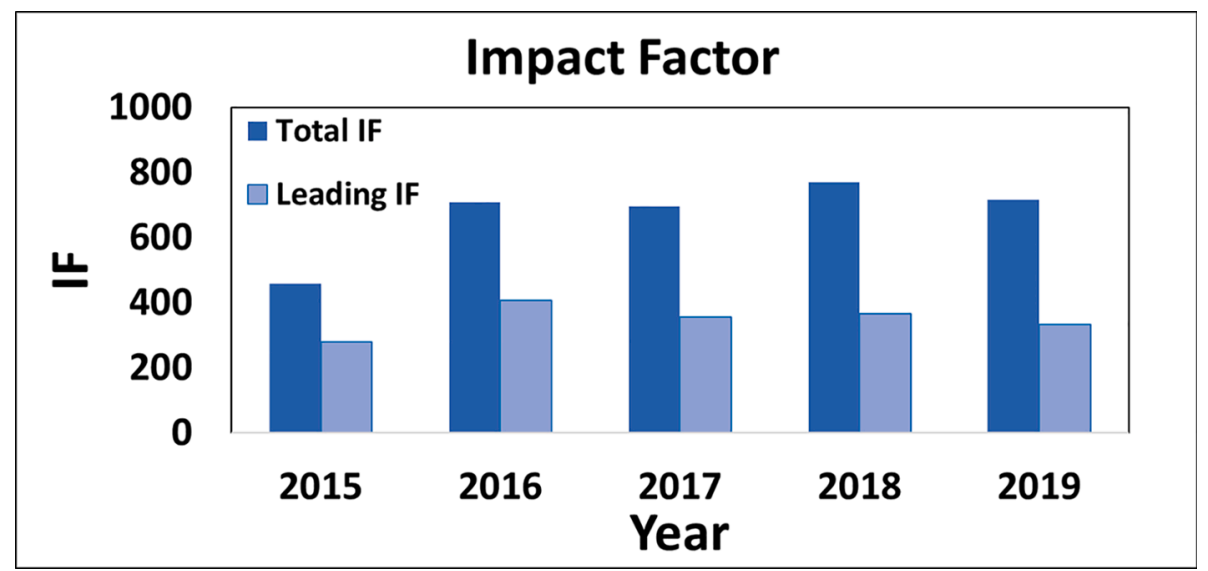

Fig. 4. Total and leading Impact Factor (IF) over the last five years, for scientific articles published by AIFM members.

articles, showing a statistically significant increase in 2016 compared to the previous year and a constant value in the next years (slightly higher than $700 / y$ ).

The journals' distribution in terms of number of published papers per journal and of papers times the corresponding journal's IF, is shown in supplementary figure 3 . The highest number of papers appeared in the following journals: Physica Medica (1 82), Physics in Medicine and Biology (64), Journal of Instrumentation (44) and Medical Physics (43). If we consider the number of papers times the IF, Physica Medica has the highest score (451), followed by Radiotherapy and Oncology (210), Physics in Medicine and Biology (194) and the European Journal of Nuclear Medicine and Molecular Imaging (144).

\section{Discussion}

We carried out a first analysis of the scientific publications of the AIFM members in the 5-year period 2015-2019, in terms of number of articles published in peer-reviewed journals. We found a total of 1210 papers, mostly in the field of Radiation Oncology (48\%), followed by Radiology (22\%), and Nuclear Medicine (16\%). The remaining 14\% of the articles were in the fields of PI, RP and Other. The distribution of published articles in the various areas could also reflect the fact that these fields are less driven by clinical needs.

A rough approximation of the weight of the Italian scientific articles' production in the field, useful in current context, may come from looking at the relative contribution of papers from Italy in medical physics journals (Scopus database). For instance, of the total number of papers published in Physica Medica (official journal of AIFM and EFOMP) in the period 2015-2019 (1196), 259 (238 original papers, 14 reviews, 4 editorials, 1 letter, 2 conference papers) included at least one Italian author (21.7\%, 1st rank position). As regards articles published in the journal Physics in Medicine and Biology, Italy contributed in the same time period a total of 186 (184 original papers, 1 review, 1 technical note) out of 3255 total papers (5.7\%, 8th rank position by country) and out of 1706 papers from European authors (10.9\%, 5th rank position by European countries) following countries with a much higher number of medical physicists with the only exception of France and the Netherlands. Finally, Italian authors contributed to the journal Medical Physics with 223 papers (205 original papers, 6 letters, 5 reviews, 4 conference papers, 2 editorials, 1 note) out of 5732 total papers (3.9\%, 10th rank position by country) and out of 1777 papers from European authors (12.5\%, 4th rank position).

Our analysis is consistent with these rough data from Scopus in confirming the impression that the scientific production of Italian medical physics is relevant in the European (and worldwide) context.

Although the majority of papers (47\%) were based on monocentric studies, a large part of the total number of articles resulted from national multicentric studies (31\%) and international collaborations $(22 \%)$, showing active interaction among the Medical Physics departments in Italy and an important commitment of the AIFM medical physics 
community at the international level, not only within Europe but worldwide, as shown in Fig. 3. To the best of our knowledge, this study represents the first initiative to quantify the scientific production of medical physicists who are members of a national scientific society.

Zeinali-Rafsanjani et al. [22] assessed the distribution and trends of studies in different areas of medical physics published on four Medical Physics journals from 2010 to 2015, identifying area that need to be more investigated in the future.

The major aim of the current survey was to provide the National Society AIFM with a tool to support the design of future activities aiming to promote and support the scientific activity of medical physicists. The result of this work by the AIFM working group FutuRuS may support future activities of AIFM Scientific and Research Committees, supporting AIFM management at promoting actions and implement policies to reinforce the scientific role of medical physicists in synergy with their professional role in agreement with the policies of international society, such as ESTRO and AAPM [16-18].

This initiative represents a pilot example for other National Societies who are willing to promote and support the scientific role of their own members with specific policies. It would be desirable that such analysis could be carried out by other European National Societies in order to promote common actions and policies at the European level towards a harmonization of the scientific and professional level of the medical physicists.

In our survey, the majority of papers in the field of RO were focused on modelling of the clinical outcome of a treatment or more in general on clinical results (27\%). This indicates a strong contribution of AIFM medical physics community to clinical studies either in performing multivariable modelling of clinical outcomes, including for example dose-volume information and clinical factors, as well as multi-omics data integration.

The second most frequent topic was particle therapy (22\%), showing that in this field the research represents an important part of the research activities of medical physics in Italy. Indeed, although only three particle therapy facilities are currently active in Italy, the research in this field is essential for a safe and efficient widespread use of this promising radiotherapy modality. Of note, most publications (91\%) were from the three Italian particle therapy centres, but also other centers that were planning to build a new particle facility contributed to this topic (9\%).

Concerning RAD, the higher percentage of publications were on dosimetry and QA (41\%), probably also due to the continuous evolution of the European legislation for radiation protection [14] that requires dose tracking and reporting in all radiological procedures. Of note, publications related to image quality and quantitative imaging/radiomic topics are expected to increase in the next years, probably more supported by AI, in particular to highlight the quantitative information encrypted in diagnostic images [23]. In NM, the highest percentage of publications were on PET (32\%), but the results for NM therapy and dosimetry (30\%) and conventional NM (26\%) were comparable, highlighting the important contribution of Italian MPEs in all the aspects of 'in vivo' nuclear medicine research, including the historical conventional areas. The topic 'Radiopharmacy and radiobiology', after an initial rise, starting from 2017 showed an important decrease in number of publications, probably due to a reduced introduction of new PET radiopharmaceuticals compared to the past.

It is interesting to highlight the low number of publications on big data/AI/Automation topic both in the RO and RAD area of interest, probably due to the fact that this topic was still not mature in the community during the investigated timeframe. On the other hand, "dosimetry and QA" as well as "Photon and high-energy electron treatment" topics in the RO area, contributed constantly with a slightly increasing number of publications over the years, showing how both topics still represent not only a clinical routine expertise but also a very productive scientific publication topic for the MPE.

The leading authorship by AIFM medical physicists was found in $56 \%$ of the papers. This means that in almost the remaining half (44\%) of the papers, the medical physicists contributed to clinical works or participated in international studies not led by themselves. In particular, within the international collaborations, the topics presenting the highest percentage of active authorship were IGRT/ART (100\%), brachytherapy (80\%) and radiopharmacy and radiobiology (80\%).

Although most papers appeared in the journal Physica Medica (15\% of the total number of papers examined), as expected, a high percentage (54\%) of the publications were on clinically oriented journals, confirming the great involvement of the MPEs in the clinical studies. Importantly, publishing not only on general Medical Physics Journals but also on clinically oriented journals (eg. Radiotherapy and Oncology, International Journal of Radiation Oncology, Biology, Physics, and European Journal of Nuclear Medicine and Molecular Imaging) allows the MPEs to increase their visibility in the medical environment with a consequent broadening of their horizons. Indeed, if we consider the number of published papers times the journal IF, as shown in Fig. S3, the impact of the higher IF of clinically-oriented and pure clinical journals (IF = 2.8-17.3) compared to that of medical physics journals ( $I F=1.5-3.2$ ) was evident.

This analysis represents a first quantitative evaluation for future trend analyses, including in particular the number of papers per year, the area of interest and their relative topics and type of the study at the basis of the paper. Moreover, it represents the basis for comparison with other countries, first within Europe. This could result in identification of common research topics to establish new international collaborations.

Looking at time-trends, apart from a significant increase between 2015 and 2016 in terms of number of papers and IF, all indicators remained stable between 2016 and 2019. To accurately explain the time trend of the number of published papers per year, a larger time interval would have been useful. Indeed, the statistically significant increase in the number of publications observed from 2015 to 2016 may be interpreted assuming a constant increase in the previous years, most likely linked to the great technological developments in all areas of Medical Physics in the past decade, followed by the observed worrying plateau from 2016. This analysis will be repeated in the following years to better assess the observed time trend. Given the global growth of publications worldwide and, in our specific fields, of the number of potential authors (due to the constant increase of the number of AIFM members), this result is a clear warning claiming AIFM for active actions to reinforce the attitude to science of its members.

As debated by others [16-18,24], the stronger assessment of the professional recognition of hospital medical physicists, also requiring new roles and responsibilities, may translate in reduced time and space for the scientific activity in medical physics services/department, especially if resources are limited. This represents a quite relevant issue especially for countries with financial resources dedicated to research and health well below the average EU standards, as Italy in the considered period.

As the scientific production is obviously a major indicator of the weight and recognition of medical physics in the field of radiological sciences, in medicine and more in general in the society, AIFM is asked to set strategies to substantially increase the scientific production of its members in the middle and long term.

In the authors' intentions, the current survey will be useful to draw the attention of the AIFM Society to specific areas of research of particular novelty and interest, either as far as education is concerned [25], establishing new teaching courses or workshop, either supporting the scientific/research activity of its members, promoting working groups or scientific collaborations with University and other research Institutes. Indeed, the interaction between the University and the Hospital environment is essential for translating the cutting-edge research performed at the University to fulfil unmet needs of the clinical practice. AIFM, gathering academic and hospital medical physicists, can play a crucial role in promoting such a collaboration.

The above presented assessment of the scientific production of AIFM 
members in the years 2015-2019 has some limitations. The Elsevier's abstract and citation database "Scopus" was interrogated using the "Scopus Author" function and a reference medical physicist for each group. This modality was chosen after having tried other research ways in "Scopus", such as by MP affiliation, which resulted difficult in paper census and unsuccessful. Using a minor number of MP groups and a reference medical physicist for each department and considering only medical Physics departments with more than 3 MPE, a number of scientific publications could have been lost by the present survey. On the other hand, the fraction of papers not registered by the survey is expected to be marginal.

The FutuRuS database containing these surveyed data is available to AIFM for further analyses and use. Its update with time will be considered to monitor the scientific production of the Society and to quantitatively compare future temporal trends.

\section{Declaration of Competing Interest}

The authors declare that they have no known competing financial interests or personal relationships that could have appeared to influence the work reported in this paper.

\section{Appendix A. Supplementary data}

Supplementary data to this article can be found online at https://doi. org/10.1016/j.ejmp.2021.06.011.

\section{References}

[1] Makropoulou M, Kareliotis G, Spyratou E, Drakaki E, Serafetinides AA, Efstathopoulos E. Non-ionizing, laser radiation in Theranostics: the need for dosimetry and the role of Medical Physics. Phys Med 2019;63:7-18.

[2] Nuesslin F, Smith P. Medical Physics now classified internationally as a profession. Med Phys 2011;38(8). i-i.

[3] Smith PHS, Nuesslin F. Benefits to medical physics from the recent inclusion of medical physisicts in the International Classification of standard occupations (ICSO-08). Med Phys Int 2013;1(1):10-4.

[4] https://www.ilo.org/public/english/bureau/stat/isco/docs/medicalphy.pdf.

[5] Delis H, Homolka P, Chapple CL, Costa PR, Attalla E, Lubis LE, et al. Developing and implementing a multi-modality imaging optimization study in paediatric radiology: experience and recommendations from an IAEA coordinated research project. Phys Med 2021;82:255-65.

[6] Delis H, Christaki K, Healy B, Loreti G, Poli GL, Toroi P, et al. Moving beyond quality control in diagnostic radiology and the role of the clinically qualified medical physicist. Phys Med 2017;41:104-8. https://doi.org/10.1016/j. ejmp.2017.04.007. Epub 2017 Apr 12.

[7] Mankoff DA, Pryma DA. The contribution of physics to Nuclear Medicine: physicians' perspective on future directions. EJNMMI Phys 2014;1(5). https://doi. org/10.1186/2197-7364-1-5. Epub 2014 May 1.
[8] Baumann M, Krause M, Overgaard J, Debus J, Bentzen SM, Daartz J, et al. Radiation oncology in the era of precision medicine. Nat Rev Cancer 2016;16(4): 234-49.

[9] Fiorino C, Guckenberger M, Schwarz M, Heide UA, Heijmen B. Technology driven research for radiotherapy innovation. Mol Oncol 2020;14(7):1500-13. https://doi, org/10.1002/mol2.v14.710.1002/1878-0261.12659.

[10] Samei E, Grist TG. Why physics in medicine? Phys Med 2019;64:319-22.

[11] Christin Knopf A, Stützer Kristin, Richter C, Rucinski A, da Silva J, Phillips J, et al. Required transition from research to clinical application: report on the 4D treatment planning workshops 2014 and 2015. Phys Med 2016;32(7):874-82.

[12] Schüller A, Heinrich S, Fouillade C, Subiel A, De Marzi L, Romano F, et al. The European Joint Research Project UHDpulse - metrology for advanced radiotherapy using particle beams with ultra-high pulse dose rates. Phys Med 2020;80:134-50.

[13] Czerska AK, Emert F, Kopec R, Langen K, McClelland JR. A Meijers et al. Clinical practice vs. state-of-the-art research and future visions: report on the 4D treatment planning workshop for particle therapy - edition 2018 and 2019. Phys Med 2021; 82.54-63.

[14] Council Directive 2013/59/Euratom of 5 December 2013 laying down basic safety standards for protection against the dangers arising from exposure to ionising radiation. https://urlsand.esvalabs.com/? $\mathrm{u}=\mathrm{https} \% 3 \mathrm{~A} \% 2 \mathrm{~F} \% 2 \mathrm{Feur}-\mathrm{lex}$. europa.eu \%2Feli\%2Fdir\%2F2013\%2F59\%2Foj\&e=000823b1\&h=d9ea60c9\& $\mathrm{f}=\mathrm{y} \& \mathrm{p}=\mathrm{n}$.

[15] Bortfeld T, Jeraj R. The physical basis and future of radiation therapy. Br J Radiol 2011;84(1002):485-98.

[16] Bortfeld T, Torresin A, Fiorino C, Andreo P, Gagliardi G, Jeraj R, et al. The research versus clinical service role of medical physics. Radiother Oncol 2015;114(3): 285-8.

[17] Fiorino C, Muren LP, Clark CH, van Elmpt W, Jornet N. Expanding the scientific role of medical physics in radiotherapy: time to act. Radiother Oncol 2015;117(3): 401-2.

[18] Ludvig P, Muren LP, Nuria Jornet N, Georg D, Garcia R, Thwaites DI. Improving radiotherapy through medical physics developments. Radiother Oncol 2015;1: 403-6.

[19] https://www.aapm.org/org/structure/?committee code=WGFRMP.

[20] Fiorino C, Jeraj R, Clark CH, Garibaldi C, Georg D, Muren L, et al. Grand challenges for medical physics in radiation oncology. Radiother Oncol. 2020 Oct 8:S01678140 (20)30837-9. doi: 10.1016/j.radonc.2020.10.001. Online ahead of print.

[21] Garibaldi C, Essers M, Heijmen B, Bertholet J, Koutsouveli E, Maas AJJ, et al. Towards an updated ESTRO-EFOMP core curriculum for education and training of medical physics experts in radiotherapy - a survey of current education and training practice in Europe. Phys Med. 2021 Apr 13;84:65-71. doi: 10.1016/j. ejmp.2021.03.030. Online ahead of print.

[22] Zeinali-Rafsanjani B, Mosleh-Shirazi MA, Saeedi-Moghadam M, Sefidbakht S. Evaluating the distribution of research in radiation sciences as published in general medical physics journals. Egypt J Radiol Nucl Med 2018;49:1119-24.

[23] Kortesniemi M, Tsapaki V, Trianni A, Russo P, Maas Ad, Källman H-E, et al. The European Federation of Organisations for Medical Physics (EFOMP) White Paper: big data and deep learning in medical imaging and in relation to medical physics profession. Phys Med 2018;56:90-3. https://doi.org/10.1016/j.ejmp.2018.11.005.

[24] Caruana CJ, Tsapaki V, Damilakis J, Brambilla M, Martín GM, Dimov A, et al. EFOMP policy statement 16: the role and competences of medical physicists and medical physics experts under 2013/59/EURATOM. Phys Med 2018;48:162-8. https://doi.org/10.1016/j.ejmp.2018.03.001.

[25] Maas AJJ, Lammertsma AA, Agius S, Bert C, Byrne B, Caruana CJ, et al. Education, training and registration of Medical Physics Experts across Europe. Phys Med 2021; 85:129-36. 